\title{
Seismic Imaging Method for Medical Ultrasound Systems
}

\author{
Daniela Theis๑* and Ernesto Bonomi® \\ Imaging and Numerical Geophysics, CRS4 (Center for Advanced Studies, Research \& Development in Sardinia), \\ 09050 Pula, Italy
}

(Received 28 January 2020; revised 28 May 2020; accepted 9 June 2020; published 9 September 2020)

\begin{abstract}
Conventional medical ultrasound systems normally implement ray-based imaging algorithms, such as delay-and-sum beamforming, whose severest limitation derives from the implicit assumption of constantvelocity media. As a result, in the case of two or more tissues with different velocities, the image of the underlying targets appears strongly degraded both in placement and in resolution. The proposed ultrasound-imaging strategy, a value-added application of concepts developed in the context of seismic prospecting, avoids this restriction by relying on the undulatory description of the physical process and not on the geometric one. Echoes are sensed in the synthetic aperture configuration by the transducer, whose elements sequentially emit a nearly spherical wave front that covers the whole region of interest. Given a macrovelocity model, the recorded echoes become the initial condition for the downward propagator of the ultrasound wavefield. The processing is in three steps: time-reverse propagation of the sensed echoes, forward simulation of the wavefield emitted by the source element, and partial imaging by computing the zero-lag temporal correlation of the two wavefields to detect the scattering structures. The final reconstruction is obtained by stacking all the partial results on a single image. Laboratory tests, performed on experimental data acquired on a physical phantom, with and without an aberrant layer, prove the effectiveness of the proposed method even in the case of vertical and lateral velocity variations, with images of impressive spatial resolution and highly accurate target placement.
\end{abstract}

DOI: 10.1103/PhysRevApplied.14.034020

\section{INTRODUCTION}

This work presents an original ultrasound-imaging strategy, operating in the frequency domain, which relies on the undulatory description of the physical process, thus differing from the simplified geometric model of most of the current ultrasound systems.

Conventional ultrasound systems for medical imaging are based on the impulse-echo technology known as beamforming [1]. An array of piezoelectric elements assembled in a transducer emits an acoustic beam focused on a target, records the backscattered echoes, and transmits them to hardware that dynamically composes them to form an image [2-5]. Appropriate delays must be used, both in transmission and in reception, to steer the acquisition along a predetermined view line. The estimate of these delays relies on the assumption that the illuminated region is made up of scattering points embedded in a uniform background

\section{*theis@crs4.it}

Published by the American Physical Society under the terms of the Creative Commons Attribution 4.0 International license. Further distribution of this work must maintain attribution to the author(s) and the published article's title, journal citation, and DOI. characterized by a sound velocity of about $1540 \mathrm{~m} / \mathrm{s}$, which is typical of soft tissues [6]. Within this idealization, each point diffracts the incident beam and gives rise to a fan of straight ray trajectories emerging at the transducer surface. For each insonified point, the potential round-trip time of the ultrasound pulse, sensed by a subset of elements around the view line, can be a priori evaluated, stored in a table, and then used to form an image by properly delaying and summing the captured signals. It is by sequentially insonifying all the view lines that the illuminated portion of the region of interest can be entirely imaged with emitted frequencies that, depending on the target depth, range anywhere between 1 and $20 \mathrm{MHz}$. Above and below the focused target, the beamformed backscattered signals are plagued by out-of-focus echoes that compromise the lateral resolution and the contrast of the image.

However, the severest limitation of beamforming comes from the implicit assumption of homogeneity of the medium, which precludes correct imaging in the presence of variable-velocity regions. The result is a degraded image with distortions caused by phase aberration [7-9].

Several authors have attempted to augment ultrasound technology with high-definition undulatory methods derived from seismic exploration [8,10-13]. The inadequacy of the results was mainly due to the zeroing of the source-to-receiver offset, a constraint imposed on 
the acquisition by Stolt's $\omega-k$ algorithm designed on the exploding-reflector model in a uniform medium [14-16].

Instead, the proposed approach starts from concepts and numerical methods that, formulated by Gazdag and Sguazzero $[17,18]$ in the context of depth seismic imaging at the dawn of supercomputing, have given rise to a consolidated theory that can provide the reconstruction of the medium without the hypothesis of homogeneity and without any constraint on the acquisition. The wave propagation is one way in depth and operates, in the Fourier domain, forward and backward in time.

The acquisition pattern that best matches the proposed imaging strategy is the synthetic aperture, where, as in geophysical prospecting, only one transducer element is used at a time to emit a nearly spherical pressure wave that illuminates the whole region of interest, while all other elements receive the backscattered signal $[11,19,20]$.

Given a macrovelocity model, the recorded echoes become the initial condition for the wavefield governed by the scalar wave equation. Each common-source gather [21] is processed in three steps [22]: time-reverse propagation of the sensed echoes [23], forward simulation of the wavefield emitted by the source element, and partial imaging by computing the zero-lag temporal correlation of the two wavefields to detect the potential scattering structures, even in the presence of vertical and lateral velocity variations. The final reconstruction is formed by stacking on a single image all the partial results obtained from common-source gathers corresponding to different source locations.

The insertion in this processing of the downward wave propagator, operating forward and backward in time, produces a simple, accurate, and potentially fast numerical engine. Very high resolution can be achieved because the numerical "illumination" of the targets comes from different angles, each of which provides a partial image that includes, in our formulation, also the accurately controlled contribution of the numerical evanescent waves.

A comparison, performed by imaging data acquired on a physical phantom with and without an aberrant layer, illustrates the advantage and the strength of the proposed method with respect to conventional delay-and-sum beamforming. The same ultrasound system (a modified Esaote MyLab 70 XVG system) and transducer (Esaote LA332) are used in the two laboratory experiments.

The outline of this article is as follows. Section II presents the undulatory model and the numerical engine of the proposed approach. Section III describes the data back propagation and the imaging process assuming a homogeneous medium, while the generalization to layered media is presented in Sec. V. In Secs. IV and VI, two laboratory tests illustrate the effectiveness of the method with and without an aberrant layer. Finally, some relevant conclusions are drawn in Sec. VII.

\section{THE ONE-WAY WAVE EQUATION}

The echo-reconstruction technique for nonintrusive imaging has wide application, from subsurface and underwater imaging to medical and industrial diagnostics. This technique is based on experiments in which a collection of short acoustic impulses, or shots, emitted at the surface illuminate the region investigated and are backscattered by the inhomogeneities of the medium, which act as reflecting interfaces causing signal echoing. The echoes are then recorded at the surface and processed through a "computational lens" defined by a propagation model to yield an image of the same inhomogeneities, which, in medical applications, are considered incorporated in an aqueous background characterized by a sound velocity $v$ of about $1540 \mathrm{~m} / \mathrm{s}$, which is close to that of soft tissues [6].

In seismic reflection applications, the recorded signals are used as initial conditions for a wavefield that must be numerically downward propagated through the illuminated medium. Typically, three-dimensional subsurface imaging, being as it is the outcome of repeated steps of seismic data acquisitions, operates on terabytes of data, which are transformed and reduced to form a meaningful image.

In comparison, the size of one complete twodimensional ultrasound synthetic aperture acquisition is hundreds of megabytes, which, with the constraint of real time, must be repeated several tens of times per second, leading to the processing of several gigabytes per second. With this strict requirement, innovation in ultrasound systems involves a simplified wave-propagation model that must, however, maintain the essential characteristics of the physics to enhance image accuracy. The resulting increased computational complexity must be balanced by the effectiveness of deployment and acceleration on high-performance-computing engines.

\section{A. Forward and backward time propagation}

An attractive answer is to combine in the Fourier domain the following two forms of the scalar one-way wave equation [24] for homogeneous media [17]:

$$
\begin{aligned}
\frac{d \widehat{P}}{d z}\left(k_{x}, z, \omega\right) & = \pm i k_{z} \widehat{P}\left(k_{x}, z, \omega\right), \\
k_{z} & =\frac{\omega}{v} \sqrt{1-\left(\frac{v k_{x}}{\omega}\right)^{2}}, \quad \omega>0,
\end{aligned}
$$

where $k_{x}$ and $k_{z}$ are the horizontal and vertical wave numbers, respectively, and $\omega$ is the angular frequency.

As in the Fourier $\left(\omega, k_{x}\right)$ domain, the full scalar wave equation can be factorized into two one-way propagators:

$$
\left(\frac{d}{d z}+i k_{z}\right)\left(\frac{d}{d z}-i k_{z}\right) \widehat{P}\left(k_{x}, z, \omega\right)=0 .
$$


It is clear that the two solutions of Eq. (1) are also the characteristic solutions of Eq. (2), which take the form

$$
\widehat{P}_{ \pm}\left(k_{x}, z+\Delta z, \omega\right)=e^{ \pm i k_{z} \Delta z} \widehat{P}_{ \pm}\left(k_{x}, z, \omega\right)
$$

These two propagators extrapolate, through a shift of phase, the two wavefields $\widehat{P}_{ \pm}\left(k_{x}, z, \omega\right)$ from depth $z$ to $z+$ $\Delta z$. Since by convention the $z$-axis points downward, the solution with the plus sign corresponds to the time-reverse propagation of the wave front, and the solution with the minus sign corresponds to the forward propagation.

With these two solutions, numerical extrapolation is then made possible through the $(x, z)$ domain discretized by a structured and regular grid with horizontal and vertical steps $\Delta x$ and $\Delta z$.

\section{B. Evanescent solutions}

The propagation mode of Eq. (3) requires $k_{z} \geq 0$, a condition illustrated in Fig. 1 (top) that is controlled by

$$
\left|\kappa_{x}\right| \leq 1, \quad \kappa_{x}=\frac{v}{\omega} k_{x}, \quad \kappa_{z}=\frac{v}{\omega} k_{z} .
$$

As a consequence, constraint (4) defines a cone in the Fourier domain $\left(\omega, k_{x}\right)$, outside of which $k_{z}$ becomes a pure imaginary number that alters the nature of the propagation. The reverse time solution decays exponentially, while the forward one diverges,

$$
\widehat{P}_{ \pm}\left(k_{x}, z+\Delta z, \omega\right)=e^{\mp\left|k_{z}\right| \Delta z} \widehat{P}_{ \pm}\left(k_{x}, z, \omega\right) .
$$

Thus, constraint (4) operates in the Fourier domain discriminating the wave-propagation modes, governed by Eq. (3), from the numerical spurious ones, governed by Eq. (5).
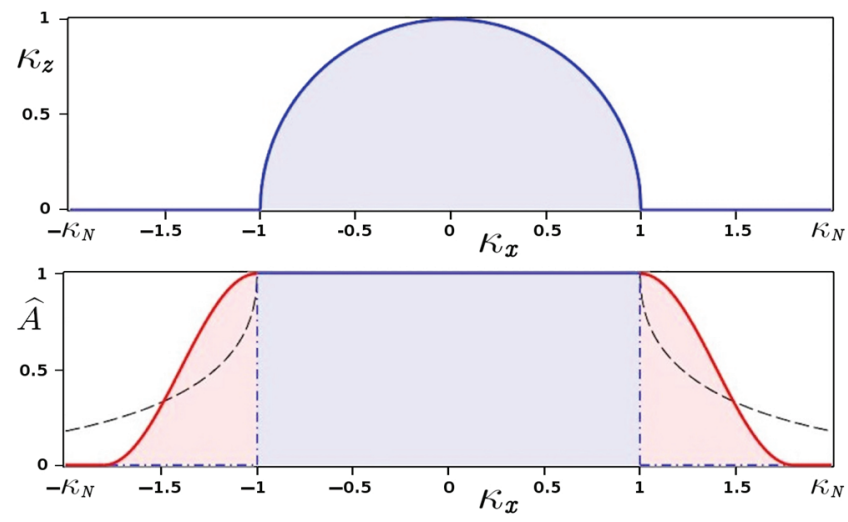

FIG. 1. Phase (top) and amplitude (bottom) of the extrapolator in the propagation zone $\left|\kappa_{x}\right| \leq 1$. Outside this zone, the dashed line corresponds to the exponential decay of the amplitude, while the solid line represents a smooth controlled decay. $\widehat{A}, \kappa_{x}$, and $\kappa_{z}$ are dimensionless quantities.
Since the proposed imaging scheme implements both solutions, the depth extrapolation outside the propagation zone must be controlled. A drastic vertical cut - that is, setting to zero the right-hand side of Eq. (5) - is, of course, to be avoided. A common approach is to continue both solutions (3) in the nonpropagating zone, making them numerically evanescent, (i.e., imposing the exponential decay along the $k_{x}$ axis). However, this exponential tapering cannot be a viable answer. Its long tail would reach the Nyquist wave number $k_{N}=\pi / \Delta x$ with strictly positive values, Fig. 1 (bottom), where $\Delta x$ denotes the spatial discretization along the $x$ axis.

Hence, the optimal continuation of Eq. (3) in the evanescent zone would be a smooth profile that avoids any sharp transition, either at $k_{x}= \pm \omega / v$ or at $k_{x}= \pm k_{N}$. More precisely, in two dimensions the curve must start with a null derivative, decay with a nonsteep slope, and fade with a null derivative, before the Nyquist wave number. An example is the cosine profile shown in Fig. 1 (bottom, solid line), where, if we set $\kappa_{N}=v k_{N} / \omega$, the evanescent amplitude takes the form

$\widehat{A}\left(\kappa_{x}\right)= \begin{cases}1 & \frac{1}{2}\left\{1+\cos \left[\frac{\pi\left(\left|\kappa_{x}\right|-1\right)}{\epsilon \kappa_{N}-1}\right]\right\} \\ 0 & \text { if }\left|\kappa_{x}\right| \leq 1, \\ \text { if } 1<\left|\kappa_{x}\right| \leq \epsilon \kappa_{N}, \\ \text { if } \epsilon \kappa_{N}<\left|\kappa_{x}\right| \leq \kappa_{N} .\end{cases}$

The width of the amplitude is controlled by $\epsilon$, chosen such that $\kappa_{N}^{-1} \ll \epsilon<1$. The design of $\widehat{A}\left(\kappa_{x}\right)$ completes the depth-extrapolation step (3), restricted by constraint (4), even in the band $1<\left|\kappa_{x}\right| \leq \kappa_{N}$, where Eq. (5) is replaced by

$$
\widehat{P}_{ \pm}\left(k_{x}, z+\Delta z, \omega\right)=\widehat{A}\left(\kappa_{x}\right) \widehat{P}_{ \pm}\left(k_{x}, z, \omega\right)
$$

All the Fourier components within the propagation cone (4) are depth extrapolated with an illumination angle $\alpha_{0}=$ $\arcsin \left(v k_{x} / \omega\right)$, while outside this cone the solution gives rise to the horizontal propagation of each $\left(\omega, k_{x}\right)$ pair, contributing to the "numerical evanescent illumination" of very steep reflectors without introducing artifacts.

In the three-dimensional case, the extension to an analytic tapering surface with the same good properties is not immediate: it must cope with the different spatial discretizations along the $x$ axis and the $y$ axis, and, notably, it must be constructed so as to avoid the angular folds typical of the Cartesian product of two curves. The ability to properly control the numerical evanescent waves is extensively underestimated $[11,12]$, despite this being a fundamental requisite for producing accurate images from the detected echoes. 


\section{IMAGING CONSTANT-VELOCITY MEDIA}

By analogy with seismic prospecting, a synthetic aperture is the acquisition configuration that naturally suits the proposed strategy for ultrasound imaging. Sequentially, each transducer element, tagged by $n$, is turned on to emit from its position $x^{(n)}$ a nearly spherical wave front that will cover the whole region of interest. The emitted front strikes the medium discontinuities, triggering reflections and diffractions that reach the transducer surface at different times. In the Fourier domain, the resulting common-source data gather $\widehat{D}^{(n)}\left(k_{x}, \omega\right)$ can then be used as a surface initial condition to regenerate the wavefield in reverse time to increase depths along the $z$ axis. The back propagation, governed by Eqs. (3) and (7), starts with $\widehat{P}_{+}^{(n)}\left(k_{x}, 0, \omega\right)=\widehat{D}^{(n)}\left(k_{x}, \omega\right)$ and provides a new $\widehat{P}_{+}^{(n)}\left(k_{x}, z, \omega\right)$ at each depth-extrapolation step.

However, the extrapolated wavefield alone is not enough to form an image of the reflecting structures. It is necessary to arrange the "handshake" with the descending wavefield emitted by the $n$th element, which provides information on the occurrence timing of all echoes subsequently collected on the transducer surface at $z=0$.

Numerically, the surface condition of the element acting as a source can be assumed as follows:

$$
\widehat{S}^{(n)}\left(k_{x}, \omega\right)=\widehat{r}(\omega) e^{i k_{x} x^{(n)}},
$$

where $\widehat{r}$ is the one-dimensional Fourier representation of the source wavelet. For example, in the case of a Ricker wavelet

$$
\widehat{r}(\omega)=\frac{2 \omega^{2}}{\sqrt{\pi} \omega_{p}^{3}} e^{-\left(\omega / \omega_{p}\right)^{2}}
$$

where $\omega_{p}$ is the most-energetic angular frequency of the spectrum. The simulation of the emitted wavefield is also modeled as the one-way depth extrapolation $\widehat{P}_{-}^{(n)}\left(k_{x}, z, \omega\right)$ of the surface condition (8); that is, $\widehat{P}_{-}^{(n)}\left(k_{x}, 0, \omega\right)=$ $\widehat{S}^{(n)}\left(k_{x}, \omega\right)$.

After the calculation of the two solutions, the imaging phase begins. At every reflecting or diffracting point at depth $z$, the arrival of the incident wavefield $\widehat{P}_{-}^{(n)}(x, z, \omega)$ must be time coincident with the back-propagated data $\widehat{P}_{+}^{(n)}(x, z, \omega)$ previously sensed by the transducer. Then, a reasonable hypothesis, devised by Claerbout [25], is that at these points a strong zero-lag temporal correlation, the aforesaid "handshake," must exist between the two wavefields. The correlation function, assembled in the angular frequency domain, becomes

$$
R^{(n)}(x, z)=\operatorname{Re}\left(\int_{0}^{\infty} d \omega \widehat{P}_{-}^{(n)}(x, z, \omega) \overline{\widehat{P}_{+}^{(n)}(x, z, \omega)}\right) .
$$

This imaging condition provides an effective reflectivity map of the portion of medium illuminated by the $n$th transducer element. Altogether, considering all the common-source gathers resulting from a complete sweep of the linear-array elements, the final image is the stack of all partial images:

$$
R(x, z)=\sum_{n=1}^{N} R^{(n)}(x, z) .
$$

The contributions of all back-propagated echoes are integrated and accumulated at every point of the image space via Eqs. (10) and (11), spanning a wide range of scattering angles between incident waves and their reflected and diffracted components. Unlike the exploding-reflector model $[12,13,15]$, where imaging considers only normalincidence rays, the focusing of this large number of events is made possible by the many source-to-receiver offsets of the synthetic aperture acquisition.

The processing of the full scan of a linear transducer with the two one-way propagators, followed by the stacking of all partial images, produces the final highresolution reconstruction. For each common-source gather, the required steps are described in Algorithm 1.

A remarkable feature of the proposed approach is the data-parallel formulation of the resulting algorithm, which can concurrently process gathers of data. Although the acquisition pattern is sequential, the method adapts well to a three-level parallelism where the computation can be organized as follows:

(a) Outer level: concurrent processing of all commonsource gathers.

(b) Middle level: simultaneous depth extrapolation of $\widehat{P}_{+}$and $\widehat{P}_{-}$.

(c) Inner level: full parallelization of the loop on the angular-frequency components $\omega$.

The final imaging [Eq. (11)] is then implemented with a reduce operation on the partial images. The memory requirement of Algorithm 1 is limited by processing only the relevant components of the spectrum; that is, $\omega_{\min } \leq$ $\omega \leq \omega_{\max }$.

To determine the computational complexity of the algorithm, in steps 1-8 let $N_{s}$ denote the number of common-source gathers, $N_{\omega}$ the number of samples in the

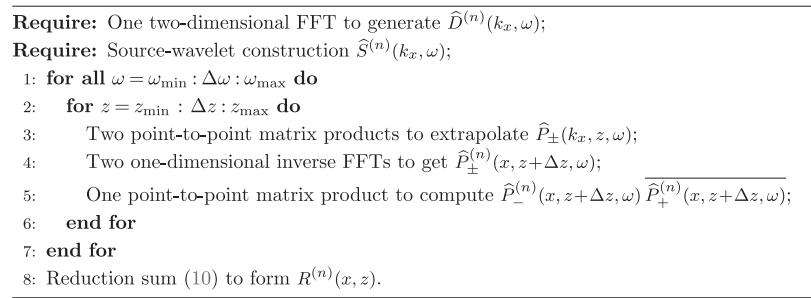

Algorithm 1. Partial imaging: constant velocity. 
range of $\omega$, and $N_{z} \times N_{x}$ the grid size of the image space. Then, the estimate of the number of floating-point operations, including FFTs [26] and point-to-point matrix operations [27], gives $C_{\mathrm{Alg} 1} \approx N_{s} N_{\omega} N_{z} N_{x}\left(10 \log _{2} N_{x}+20\right)$. In comparison, the complexity of the delay-and-sum algorithm for the whole image goes like $O\left(N_{z} N_{x}^{2}\right)$, a saving that does, however, cost the loss of accuracy of the reconstruction.

The data-parallel implementation of Algorithm 1 can concurrently process gathers of data (outer level) and angular frequencies (inner level), with an actual speedup of $N_{s} N_{\omega}$ on multiprocessor and multicore systems. Nevertheless, a further speedup factor can be gained by fully using the hardware acceleration with application-specific processing elements and overflowing computing power [28].

\section{PHANTOM-ECHO-DATA PROCESSING}

A test is performed on experimental raw data acquired in the synthetic aperture mode, with a single element transmitting and all elements receiving in a repeating cycle. The data are recorded by illuminating the $3.5 \times 10 \mathrm{~cm}^{2}$ vertical region shown in Fig. 2 of the Computerized Imaging Reference Systems (CIRS) physical phantom 040GSE, which includes grayscale hyperechoic targets and groups of nylon monofilaments of distinct diameters, embedded at different depths in an aqueous solution with sound velocity $v=$ $1540 \mathrm{~m} / \mathrm{s}$. The phantom attenuation is $0.5 \mathrm{~dB} /(\mathrm{MHz} \mathrm{cm})$. The characteristic features of each acquisition [29] are a 144 -element linear transducer with a $0.245-\mathrm{mm}$ interelement spacing, a sampling frequency of $50 \mathrm{MHz}$, and a one-cycle source pulse at $7.14 \mathrm{MHz}$ with a pulse length of $0.21 \mathrm{~mm}$. In the conventional experiment, the focus of the ultrasound beamformer is tuned to $30 \mathrm{~mm}$ (i.e., the depth of the hyperechoic target from the phantom surface).

To illustrate the advantage and the strength of the proposed method, the resulting depth imaging is compared with delay-and-sum beamforming performed on the same region by the conventional ultrasound system. Incidentally, the depth-imaging result is obtained without any preprocessing on the raw data and the only postprocessing is limited to the computation of the absolute value of the Hilbert-transformed image with a gain compensation and to the threshold of its $\log$ compression.

The clear qualitative difference and improvement are shown in Fig. 3, where the dynamic range in both images is $50 \mathrm{~dB}$. First of all, with the synthetic aperture acquisition, a wider angle of illumination is achieved because Algorithm 1 correlates and accumulates contributions from different directions, allowing the formation of the image beyond the region delimited by the view lines of conventional beamforming. For instance, the second grayscale target and the two other nylon monofilaments, at different depths, appear on the right in a position out of reach

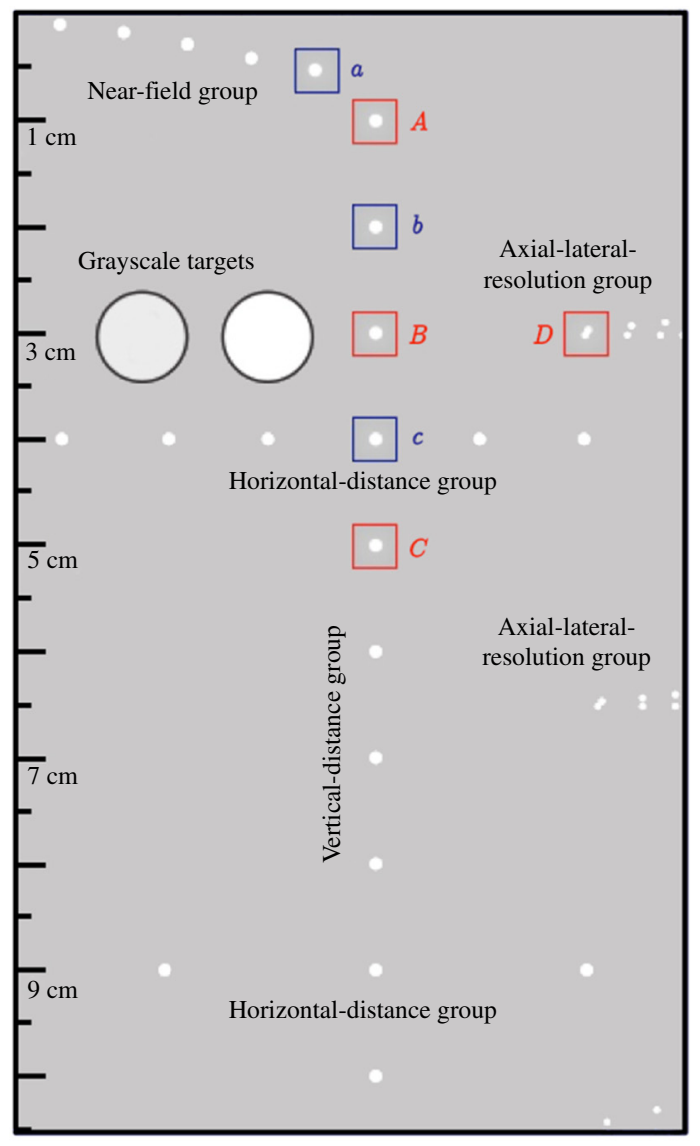

FIG. 2. Portion of the CIRS physical phantom 040GSE used for the tests, with grayscale hyperechoic targets and groups of nylon monofilaments of distinct diameters.

of conventional beamforming. Similarly, two axial-lateralresolution groups and two other nylon monofilaments appear in the dark side on the left.

Problems with conventional ultrasound systems also include low contrast and resolution outside the transmit focus zone and a lowering of the signal-to-noise ratio (SNR) with depth. Conversely, as shown in Fig. 3, the contrast and the spatial resolution of the proposed method (bottom) are overall much higher and the differences are even more remarkable as depth increases, resulting in a more-uniform and narrower appearance of the reflectors throughout the image, despite the energy decay caused by both the geometric spread of the wave front and by the attenuating action of the illuminated medium.

\section{A. Target contrast-to-noise ratio}

For a quantitative comparison, since complete knowledge of the hard-coded processing chain of the ultrasound system is not available, only the final log-compressed values of the pixels can be used. In this regard, the two images in Fig. 4, showing close-ups of the hyperechoic target reconstructions in Fig. 3, are considered. Although no clear 

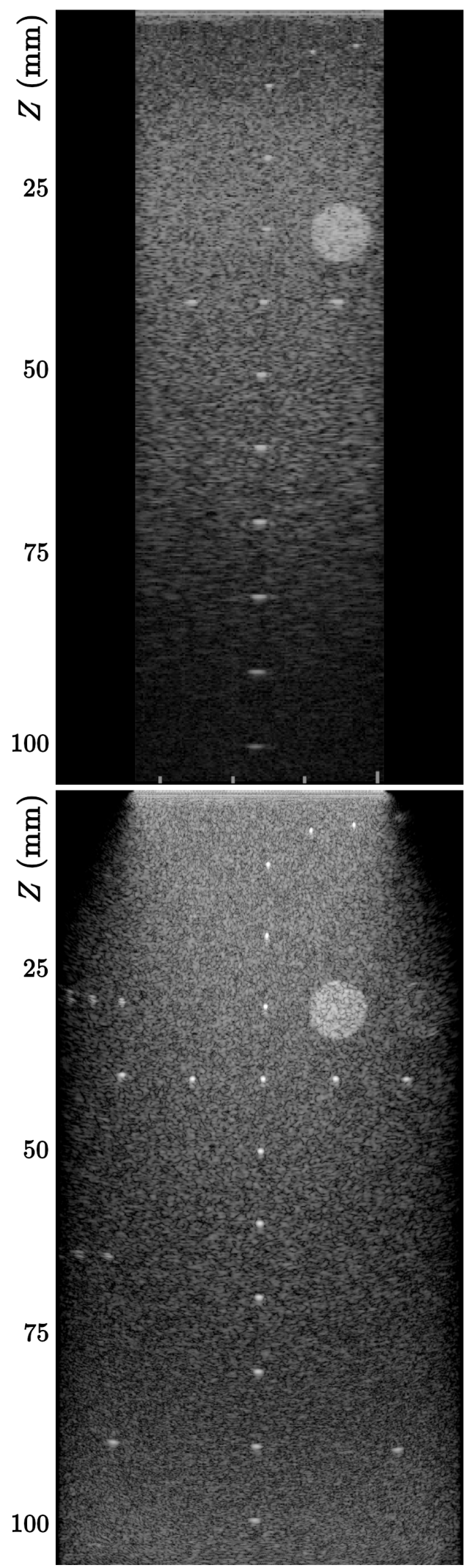

FIG. 3. CIRS physical phantom 040GSE: standard delay-andsum beamforming output of the ultrasound system (top) and depth imaging of synthetic aperture data (bottom). Both images are displayed on a dynamic range of $50 \mathrm{~dB}$.

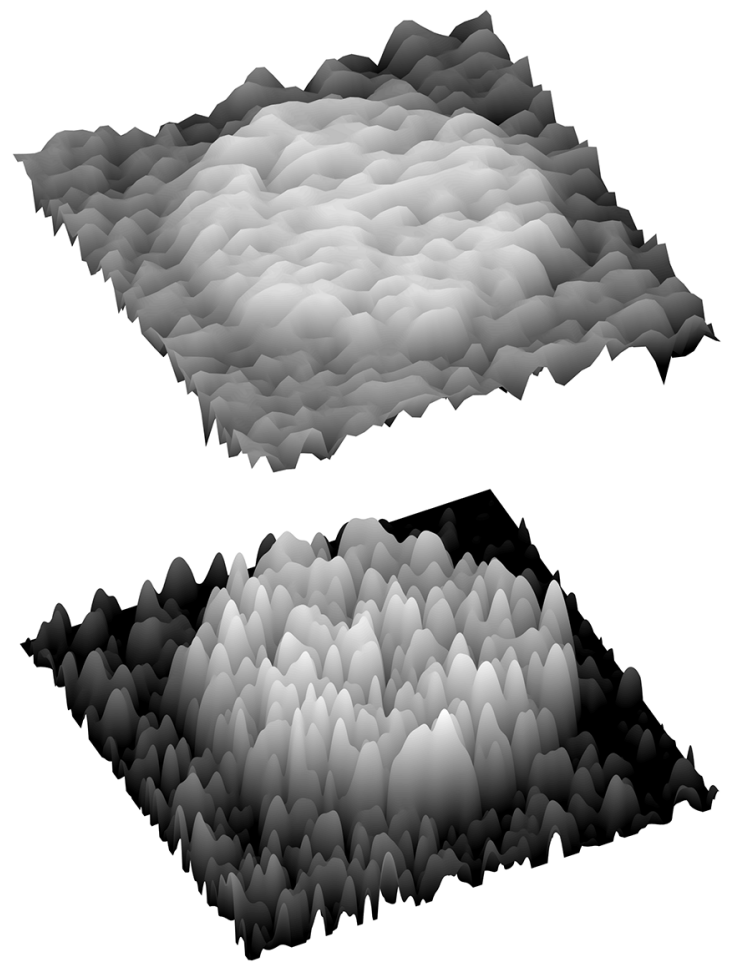

FIG. 4. Images of the hyperechoic target located at $30 \mathrm{~mm}$ (diameter $8 \mathrm{~mm}$ ): delay-and-sum beamforming output (top) and depth imaging of synthetic aperture data (bottom). The second image exhibits better gray contrast; that is, $C N R_{\mathrm{LC}}=2.16$ (top) and $C N R_{\mathrm{LC}}=4.35$ (bottom).

consensus exists on how to measure the contrast of ultrasound images [30], the contrast-to-noise ratio $\left(C N R_{\mathrm{LC}}\right)$ computed for the final log-compressed images is chosen to quantify the target's "detectability" [31]:

$$
C N R_{\mathrm{LC}}=\frac{\left|\mu_{\mathrm{ROI}}-\mu_{\mathrm{bck}}\right|}{\sigma_{\mathrm{bck}}} .
$$

In Eq. (12), $\mu$ and $\sigma$ designate, respectively, the average and the standard deviation of the pixel values, evaluated inside the selected target (ROI) and outside the target (bck) - that is, in the background noise - at the same depth [32]. The target and background regions have the same surface area. The estimate of the contrast-to-noise ratio on the hyperechoic target points to a $101 \%$ increase, which is clearly visible in Fig. 4, to the advantage of Algorithm 1, $C N R_{\mathrm{LC}}=4.35$, with respect to the ultrasound-system output, $C N R_{\mathrm{LC}}=2.16$.

\section{B. Filament and speckle signal-to-noise ratio}

Unlike the contrast-to-noise ratio, it is recommended to evaluate the SNR,

$$
S N R=\frac{\mu_{\mathrm{ROI}}}{\sigma_{\mathrm{bck}}}
$$




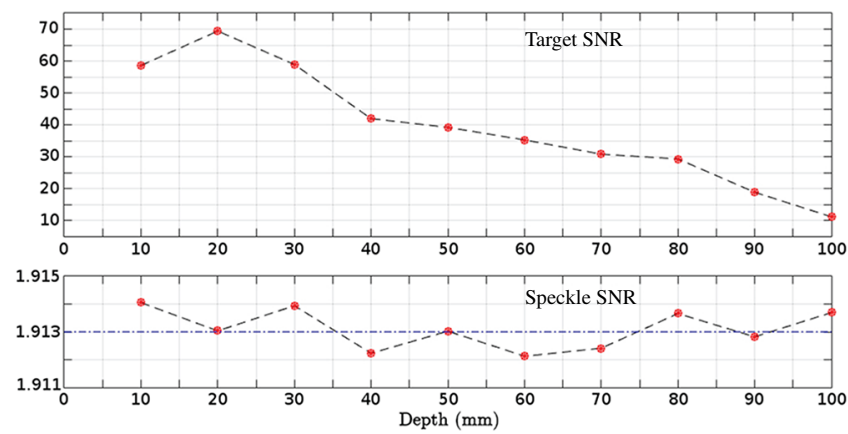

FIG. 5. SNRs of the vertical-distance-group filaments (top) and of the surrounding speckle (bottom) at the same depths $\left(S N R_{0} \approx 1.913\right)$.

on the image before compression [31]. As the exact Hilbert-transformed intensity data cannot be retrieved from the ultrasound-system output, to qualify the synthetic aperture imaging of Algorithm 1, SNR evaluations are performed with reference to a theoretical formulation on the statistics of the speckle intensity. More precisely, the mathematical model of the coherent accumulation of the random scatterings, triggered by the impinging wave front within a resolution cell of the phantom background, leads to the pixel intensity distribution according to Rayleigh's probability density function [33]. The resulting speckle signal-to-noise ratio predicted by this idealization is given by $S N R_{0}=[\pi /(4-\pi)]^{1 / 2} \approx 1.913$.

As shown in Fig. 5, the experimental estimates of the SNR are performed on the ten nylon monofilaments of the vertical-distance group and the surrounding speckle. In particular, the speckle SNRs are, at each depth, reassuringly close to the theoretical $S N R_{0}$. Moreover, the sequence of SNR measurements plotted in Fig. 5 (top) for the vertical filaments, although progressively decaying, exhibits values much higher than $S N R_{0}$, confirming that in this test the penetration depth of Algorithm 1 (i.e., the depth at which a target becomes totally overwhelmed by the speckle) is greater than $100 \mathrm{~mm}$.

\section{Filament spatial resolution}

The ability of Algorithm 1 to focus the back-propagated echoes on the position of the wires is shown in Fig. 6, and can be quantified by the full-width-at-half-maximum cutting (FWHM) of the normalized intensity. This figure illustrates the resolution capacity of the method on the first, third, and fifth wires (diameter $0.1 \mathrm{~mm}$ ) of the verticaldistance group (targets A, B, and C in Fig. 2), where each FWHM cutting is highlighted by the black contour line. The spatial resolution in the dark area of the image, on the left side of the illumination cone, is still good. Even in these extreme conditions of illumination, the resulting normalized intensity of target D, shown in Fig. 7, exhibits the clear separation between the two wires (diameter $0.08 \mathrm{~mm}$ )
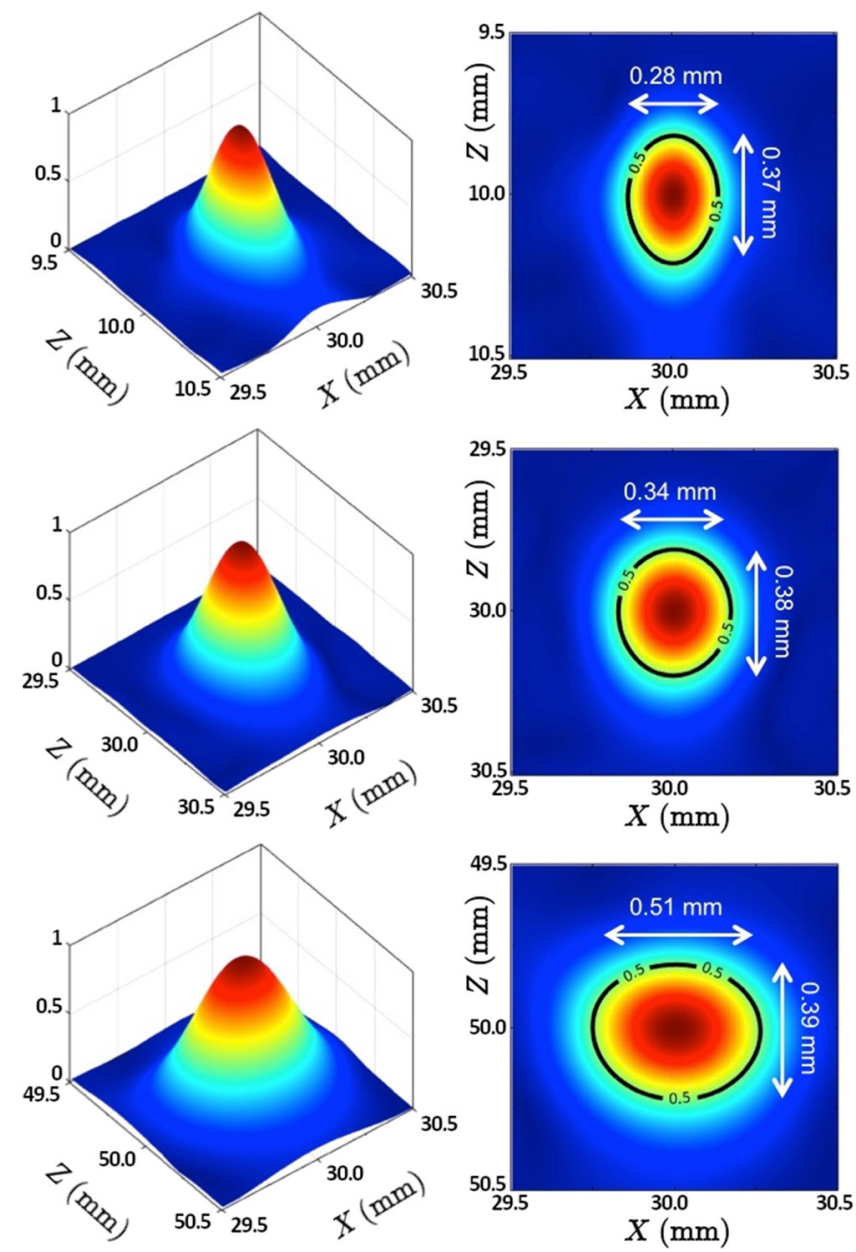

FIG. 6. Vertical-distance group: wires A, B, and C, respectively, at $1.0,3.0$, and $5.0 \mathrm{~cm}$ from the top. The FWHM of the normalized intensity allows us to quantify the spatial resolution.

that form the closest pair of the group. The obvious deformation to the left of the two bodies is caused by the finite offset of the transducer and, thus, by the lack of ultrasound data. Nevertheless, the axial resolution is notable.

\section{IMAGING THROUGH SCATTERING LAYERS}

Phase aberration is a major issue for image degradation in conventional ultrasound systems, as the imaging model they implement idealizes the medium as an assembly of diffracting points embedded in a uniform background. From this quite strong conjecture it follows that the time delays to focus the acoustic beams and those to align the received echoes and form an image are tabulated assuming a constant-velocity medium. Phase aberration thus originates from the simplification of the "computational lens," which introduces gross errors when inhomogeneous materials are present. Consequently, some additional processing is necessary to reduce the inaccuracies [7-9,34]. 

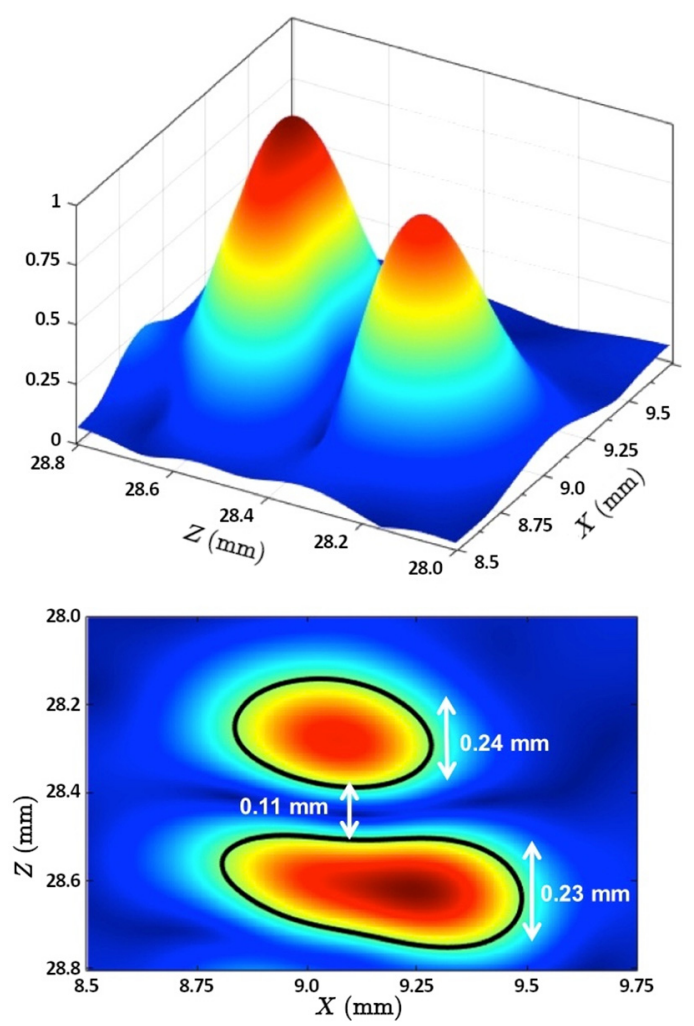

FIG. 7. Top axial-lateral-resolution group: the two closest wires, on the left, dark side, are resolved as separate entities. The FWHM of the normalized intensity allows us to quantify the spatial resolution.

The one-way wave extrapolator (3)-(7) goes beyond the limit of constant-velocity media. It can propagate the transducer surface condition, without compromising the kinematics, across vertically varying velocity fields $v=$ $v(z)$. Moreover, this constraint is overcome by adapting the depth extrapolation to more-complex media with lateral velocity variations [18]. The starting point is the phaseshift formula (3) modified to handle the wave propagation inside a layer $[z ; z+\Delta z]$, characterized by a laterally variable velocity $v=v_{z}(x)$, taking $M(z) \geq 1$ known values, $v_{z}^{(1)}<v_{z}^{(2)}<\cdots<v_{z}^{(M)}$, derived from the estimate of the macrovelocity model of the medium [35].

For stratified media, the generalized imaging algorithm takes a new form that can be synthesized according to the following computational stages:

(a) Vertically extrapolate $\widehat{P}_{ \pm}(x, z, \omega)$ - that is, the Fourier transform of $P(x, z, t)$ with respect to $t$-as follows:

$$
\widehat{Q}_{ \pm}(x, z, \omega)=\widehat{P}_{ \pm}(x, z, \omega) e^{i \omega / v_{z}(x)} .
$$

(b) Compute the horizontal correction for $v_{z}^{(m)}$ by applying the phase-shift formula (3) to $\widehat{Q}_{ \pm}\left(k_{x}, z, \omega\right)$ with

$$
k_{z}^{(m)}=\frac{\omega}{v_{z}^{(m)}} \sqrt{1-\left(\frac{k_{x}}{\omega} v_{z}^{(m)}\right)^{2}}-\frac{\omega}{v_{z}^{(m)}},
$$

and controlling the evanescent modes with Eq. (7) to get $\widehat{P}_{ \pm}^{(m)}\left(k_{x}, z+\Delta z, \omega\right)$.

(c) For all $x$ where $v_{z}(x)=v_{z}^{(m)}$, set

$$
\widehat{P}_{ \pm}(x, z+\Delta z, \omega)=\widehat{P}_{ \pm}^{(m)}(x, z+\Delta z, \omega) .
$$

(d) Repeat the depth extrapolation (16) for the $M$ reference velocities.

Processing the full scan of a linear transducer and stacking all the partial results with Eq. (11) produces the final highresolution image. Algorithm 2 describes the required steps for each common-source gather.

Algorithm 2 makes imaging feasible even with slant and uneven interfaces consisting of different materials or tissues. In comparison, a geometrically based formulation [19] would require the computation of all the traveltimes to get the adequate focusing for each inhomogeneity of the model, in both transmission and reception. Against an impracticable delay-and-sum algorithm for inhomogeneous media, Algorithm 2 effectively leads to good results by running a phase shift for each reference velocity at a computational cost slightly higher than $C_{\mathrm{Alg} 1}$.

More precisely, if we set $N_{v}=\max _{z}\{M(z)\}$, an overestimated value for the computational cost is $C_{\mathrm{Alg} 2} \approx$ $N_{s} N_{\omega} N_{z} N_{x}\left[\left(N_{v}+1\right)\left(10 \log _{2} N_{x}+12\right)+8\right]$, where $N_{v}$ is on the order of a few units.

On multiprocessor and multicore systems, Algorithm 2 inherits the good data-parallel structure of Algorithm 1 with an actual speedup of $N_{S} N_{\omega}$. Moreover, a further

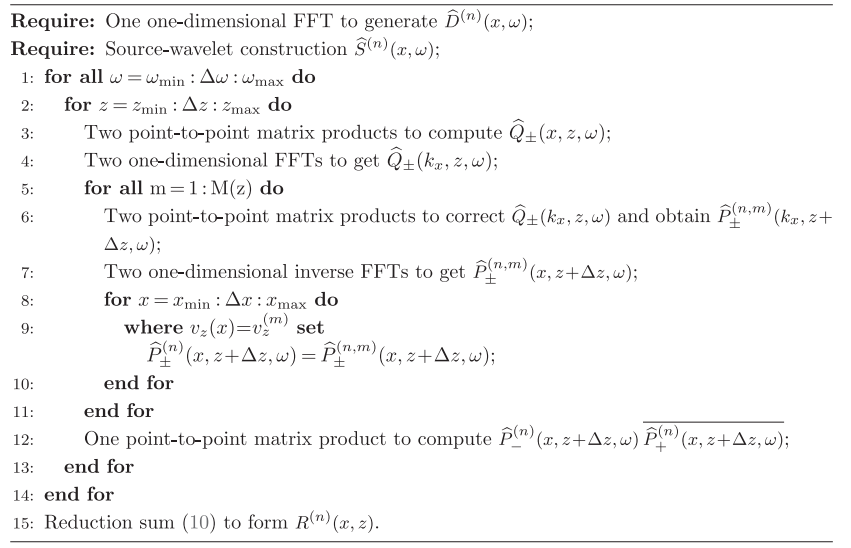

Algorithm 2. Partial imaging: variable velocity. 
speedup factor can be gained by properly fitting the programming paradigm supported by hardware accelerators and dataflow engines [28].

\section{PHANTOM TEST WITH AN ABERRANT LAYER}

As an example with two global velocity values, consider the eloquent case of the CIRS phantom, shown in Fig. 2, with on top an aberrant silicone rubber layer about $2.2 \mathrm{~cm}$ thick with a slightly slant section. Its sound velocity is around $1100 \mathrm{~m} / \mathrm{s}$, which is incidentally well outside the bounds of any possible value in human tissues [6]. The background velocity of the physical phantom, $1540 \mathrm{~m} / \mathrm{s}$, is assumed to be exact.

The top panel in Fig. 8 displays the conventional output of the ultrasound machine. Phase aberration is evident and is caused by the delay-and-sum beamforming that, not being able to cope with velocity contrasts, cannot focus the ultrasound echoes at the correct vertical and lateral positions. Therefore, because of the erroneous assumption of the same velocity of $1540 \mathrm{~m} / \mathrm{s}$ for both layers, the echoes are collocated at higher wrong depths, greatly affecting also the lateral resolution of the imaged targets. It is also worth noting the resulting excessively high amplitude of the reverberation generated within the aberrant layer by the multiple reflections of the acquired signal.

To prove the versatility of the proposed method, the depth and profile of the slant boundary between the two domains are numerically evaluated by running Algorithm 2 with $v_{z}(x)=1100 \mathrm{~m} / \mathrm{s}$, reaching the highly accurate estimate displayed in Fig. 9. While the lateral resolution of the diffractors beneath the aberrant layer obviously suffers from the choice of the velocity, the interface echoes are definitely placed at the correct depth.

The resulting macrovelocity model $v_{z}(x)$ has $M(z)=1$ with $1100 \mathrm{~m} / \mathrm{s}$ above the highest point of the interface, $M(z)=1$ with $1540 \mathrm{~m} / \mathrm{s}$ below the lowest point of the interface, and $M(z)=2$, with $v_{z}^{(1)}=1100 \mathrm{~m} / \mathrm{s}$ and $v_{z}^{(2)}=$ $1540 \mathrm{~m} / \mathrm{s}$ in between.

In general and from a practical point of view, a minor deviation of ultrasound velocities from the true values for the layers will not significantly affect the result of estimating the interface distribution [36].

Algorithm 2, driven by $v_{z}(x)$, forms the image shown at the bottom of Fig. 8 within a dynamic range of $50 \mathrm{~dB}$ : no aberration artifacts are present when the synthetic aperture data acquired on the modified phantom are depth extrapolated. The remarkable result is that, in spite of the aberrant layer, the stacking of all partial images, one for each common-source gather, collapses the ultrasound echoes not only to the proper position inside the physical phantom but also with a striking spatial resolution. It must be clear that the absence of aberration is not a postprocessing effect but is an intrinsic feature of the wave-equation-based
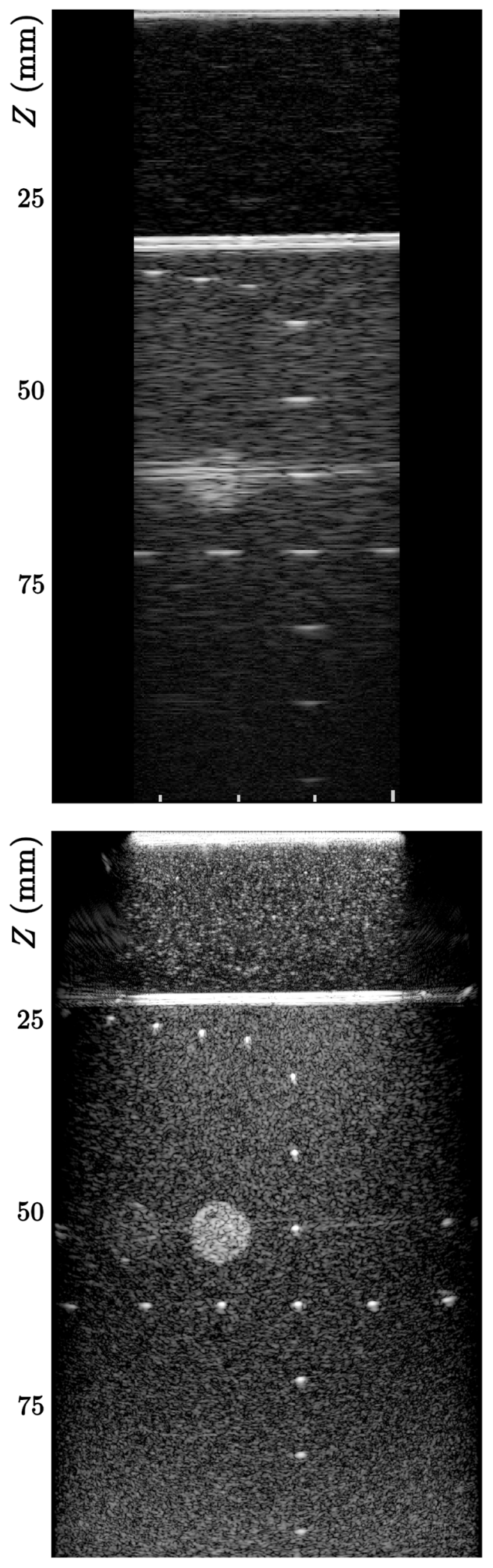

FIG. 8. CIRS physical phantom 040GSE with a slant aberrant layer on top: standard delay-and-sum beamforming output of the ultrasound system (top) and depth imaging by phase shift of synthetic aperture data (bottom). Both images are displayed on a dynamic range of $50 \mathrm{~dB}$. 


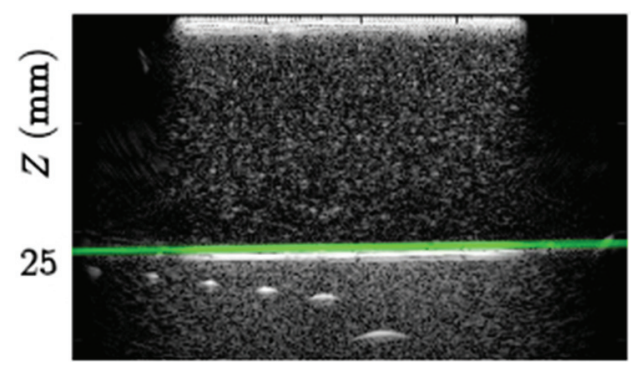

FIG. 9. Estimate of the thickness of the aberrant silicone layer obtained by running Algorithm 2 with $v_{z}(x)=1100 \mathrm{~m} / \mathrm{s}$, from 0 to $3 \mathrm{~cm}$.

imaging; namely, its ability to handle variable velocity fields.

\section{A. Target contrast-to-noise ratio}

The experimental estimate of the contrast-to-noise ratio, by application of Eq. (12) on the log-compressed hyperechoic target image, points to a $92 \%$ increase, which is clearly visible in Fig. 10, to the advantage of Algorithm 2,

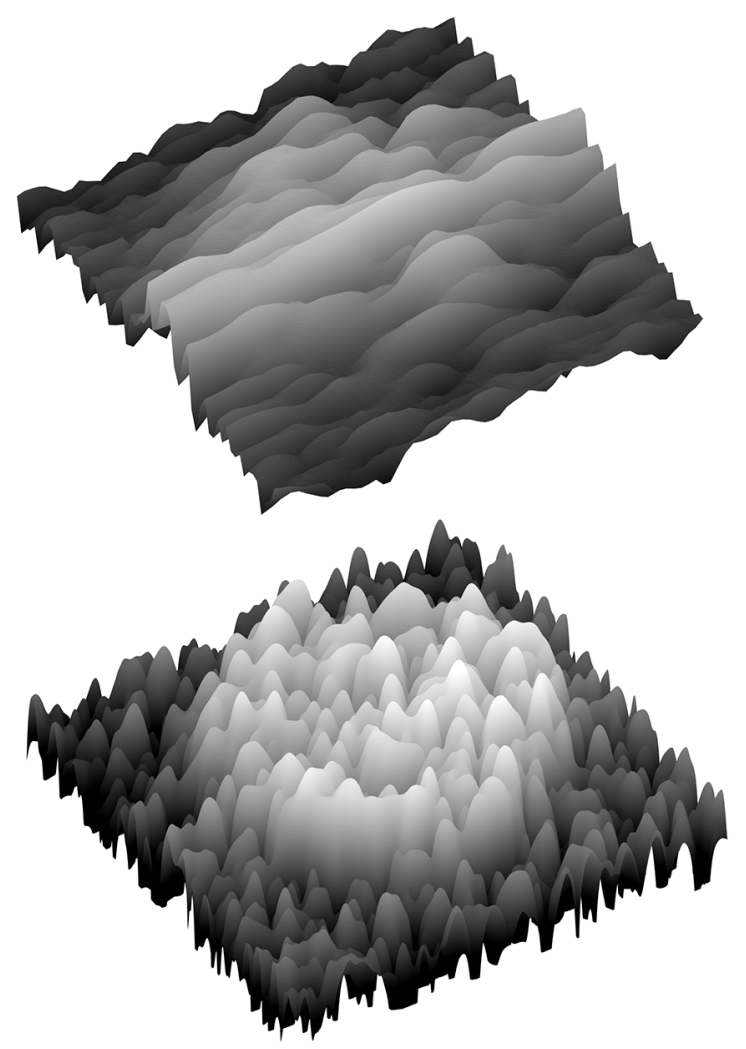

FIG. 10. Hyperechoic target located $30 \mathrm{~mm}$ below the slant aberrant layer: beamformed output (top) and depth imaging of synthetic aperture data (bottom). The lower image exhibits greater gray contrast, $C N R_{\mathrm{LC}}=1.40$ (top) and $C N R_{\mathrm{LC}}=2.69$ (bottom) and a considerable weakening of the unwanted footprint of multiple reflections.
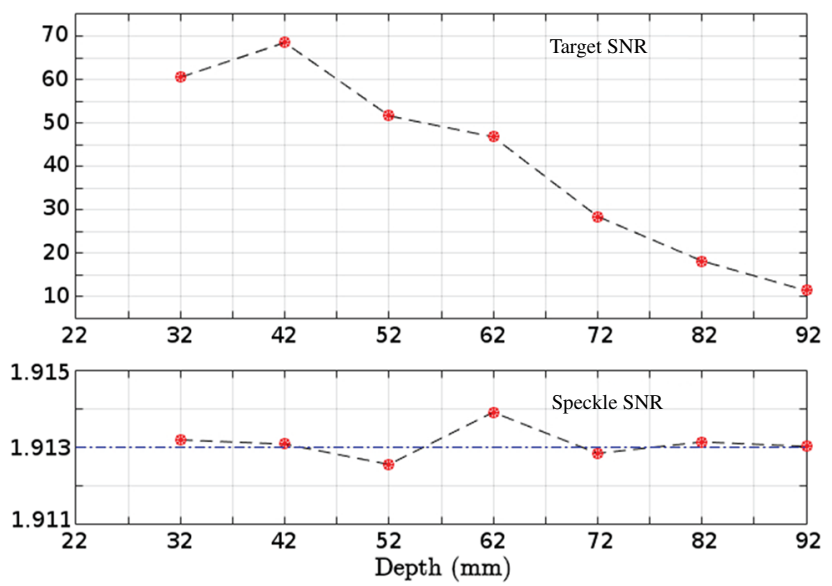

FIG. 11. SNRs of the vertical-distance group filaments (top) and of the surrounding speckle (bottom) at the same depths $\left(S N R_{0} \approx 1.913\right)$.

$C N R_{\mathrm{LC}}=2.69$, with respect to the ultrasound system output, $C N R_{\mathrm{LC}}=1.40$. This last value is calculated by excluding the spurious amplitude contribution of the multiple reflections caused by the aberrant-layer interface.

\section{B. Filament and speckle signal-to-noise ratio}

The experimental estimates of the signal-to-noise ratio are performed by applying Eq. (13) on the image before compression (i.e., on the Hilbert-transformed intensity) for the first seven nylon monofilaments of the vertical-distance group and the surrounding speckle. As shown at the bottom of Fig. 11, the speckle SNRs are, at each depth, once again reassuringly close to the theoretical $S N R_{0} \approx 1.913$, that is, the signal-to-noise ratio predicted by the idealization of the speckle random-scattering process [33]. Moreover, the sequence of SNR measurements plotted at the top of Fig. 11 for the vertical filaments exhibits, at the same depths, values that are consistent with those without an aberrant layer plotted in Fig. 5.

\section{Filament spatial resolution}

Figure 12 illustrates the resulting resolution on the target filaments a, b, and c in Fig. 2, which is particularly good despite the aberrant layer. The additive layer, with a mean thickness of $2.2 \mathrm{~cm}$, collocates the actual filament positions at a greater depth (i.e., 2.7, 4.2, and $6.2 \mathrm{~cm}$ ) than those indicated in Fig. 2. Moreover, the absence of aberration effects leads to the sharp focusing of the multiple reflections occurring within the silicone layer and the consequent weakening of their undesirable footprint, as seen in Figs. 8 and 10. 

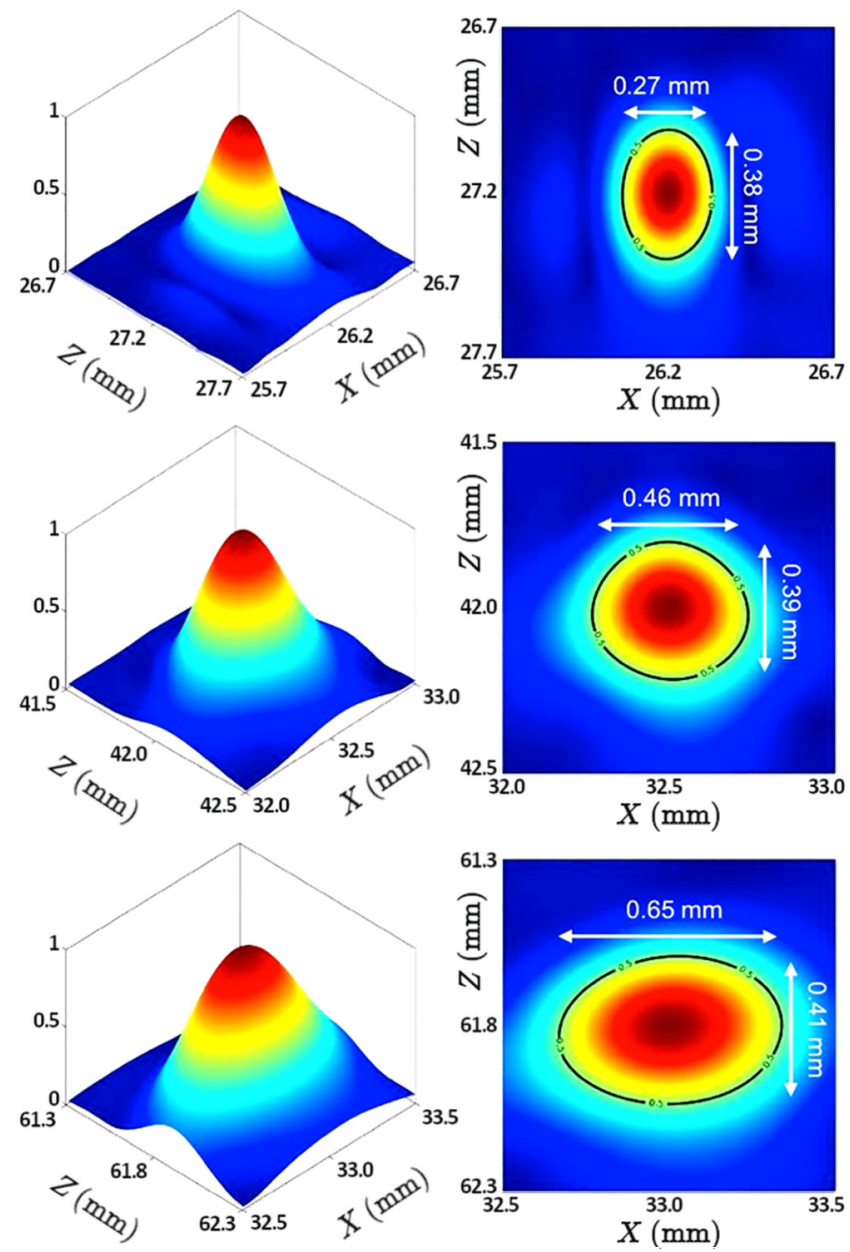

FIG. 12. Near-field group and vertical-distance group: filaments a, b, and c, respectively at $2.7,4.2$, and $6.2 \mathrm{~cm}$ from the top. The FWHM of the normalized intensity allows us to quantify the spatial resolution.

\section{CONCLUSION}

We describe an ultrasound-imaging strategy relying on the undulatory description of the acoustic propagation, which is demonstrated to be superior to the ray-based echoreconstruction approach typical of conventional ultrasound systems. The use in the Fourier domain of the one-way depth propagation of the transducer's data, together with the controlled contribution of the numerical evanescent waves, yields a very-high-resolution reconstruction.

The synergy with the synthetic aperture acquisition allows the target region to be illuminated from a wide range of incidence directions, giving rise to many overlapping partial images each containing the high-accuracy properties of the undulatory approach. As an alternative, keeping the three-step approach and its undulatory nature unchanged, nothing would prevent the adaptation of the source term of the forward problem to the steered emission of all transducer elements and the obtaining of a very fast plane-wave imaging tool $[4,12,37]$, provided one accepts a lower resolution [38].

Laboratory tests assess the effectiveness of the method described, even in the presence of an aberrant layer, and show a remarkable spatial resolution. The same algorithm can also be successfully used to estimate the interface distribution in stratified media of known tissues [6,36], thus allowing better numerical focusing of soft targets beneath multiple strata such as epidermis $(1540 \mathrm{~m} / \mathrm{s})$, dermis $(1580 \mathrm{~m} / \mathrm{s})$, fat $(1440 \mathrm{~m} / \mathrm{s})$, and muscles $(1588 \mathrm{~m} / \mathrm{s})$.

The extension of Algorithms 1 and 2 from two dimensions to three dimensions is feasible, provided one designs a good tapering surface to control the optimal damping of the evanescent propagation in the Fourier domain. Our three-dimensional implementation is already complete and satisfies this requirement. Its specialization for ultrasound imaging is currently in progress.

\section{ACKNOWLEDGMENT}

The authors thank Esaote SpA for useful technical support.

[1] W. R. Hendee and E. R. Ritenour, Medical Imaging Physics (Wiley-Liss, Inc., New York, USA, 2002), 4th ed., Chap. 19-22.

[2] S. C. Wooh and Y. Shi, Optimization of Ultrasonic Phased Arrays (Springer, Boston, MA, 1998), p. 883.

[3] J. H. Lee and S. W. Choi, A parametric study of ultrasonic beam profiles for a linear phased array transducer, IEEE Trans. Ultrason. Ferroelectr. Freq. Control 47, 644 (2000).

[4] M. Tanter and M. Fink, Ultrafast imaging in biomedical ultrasound, IEEE Trans. Ultrason. Ferroelectr. Freq. Control 60, 102 (2014).

[5] M. Toulemonde, Ph.D. thesis, School École Doctorale: Mécanique, Énergétique, Génie Civil, Acoustique, Université Claude Bernard Lyon 1, France, 2014.

[6] Tissue properties database: Speed of sound, Portal of the IT'IS Foundation, (Zurich, Switzerland, 2020), https:// itis.swiss/virtual-population/tissue-properties/database/aco ustic-properties/.

[7] L. A. Ødegaard, Ph.D. thesis, School Department of Mathematical Sciences, NTH, Norwegian Institute of Technology University of Trondheim, 1995.

[8] M. A. Haun, Ph.D. thesis, School Graduate College, University of Illinois at Urbana-Champaign, 2003.

[9] T. Varslot, Ph.D. thesis, School Department of Mathematical Sciences, NTNU, Norwegian University of Science and Technology, Trondheim, Norway, 2004.

[10] N. R. Goulding, J. D. Marquez, E. M. Prewitt, T. N. Claytor, B. R. Nadler, and L. Huang, Ultrasonic Imaging Techniques for Breast Cancer Detection, Tech. Rep. LA-UR-06-6193 (Los Alamos National Laboratory, Los Alamos, NM 87545, USA, 2007).

[11] M. H. Skjelvareid, T. Olofsson, Y. Birkelund, and Y. Larsen, Synthetic aperture focusing of ultrasonic data 
from multilayered media using an omega-k algorithm, IEEE Trans. Ultrason. Ferroelectr. Freq. Control 58, 1037 (2011).

[12] D. Garcia, L. L. Tarnec, S. Muth, E. Montagnon, J. Porée, and G. Cloutier, Stolt's f-k migration for plane wave ultrasound imaging, IEEE Trans. Ultrason. Ferroelectr. Freq. Control 60, 1853 (2013).

[13] H. J. Vos, P. L. van Neer, M. M. Mota, M. D. Verweij, A. F. van der Steen, and A. W. Volker, F-k domain imaging for synthetic aperture sequential beamforming, IEEE Trans. Ultrason. Ferroelectr. Freq. Control 63, 60 (2016).

[14] R. H. Stolt, Migration by fourier transform, Geophysics 43, 23 (1978).

[15] J. F. Claerbout, Imaging the Earth's Interior (WileyBlackwell, Palo Alto, 1985), Chap. 1.

[16] R. Stolt and A. B. Weglein, Seismic Imaging and Inversion: Application of Linear Inverse Theory (Cambridge University Press, New York, USA, 2012).

[17] J. Gazdag, Wave equation migration with the phase-shift method, Geophysics 43, 1342 (1978).

[18] J. Gazdag and P. Sguazzero, Migration of seismic data by phase shift plus interpolation, Geophysics 49, 124 (1984).

[19] J. A. Jensen, S. I. Nikolov, K. L. Gammelmark, and M. H. Pedersen, Synthetic aperture ultrasound imaging, Ultrasonics 44, e5 (2006).

[20] I. Trots, A. Nowicki, M. Lewandowski, and Y. Tasinkevych, Synthetic Aperture Method in Ultrasound Imaging (InTech, Janeza Trdine 9, 51000 Rijeka, Croatia, 2011), 3rd ed., Chap. 3, p. 37.

[21] A set of recorded signals sharing the same source element.

[22] B. L. Biondi, 3D Seismic Imaging, Investigations in Geophysics (Society of Exploration Geophysicists, Tulsa, Oklahoma, USA, 2006).

[23] All common-source signals are time reversed and numerically reinjected into the medium from the recording locations [39].

[24] In the Fourier domain, $\hat{P}\left(k_{x}, z, \omega\right)$ indicates the transformed hydrostatic pressure $P(x, z, t)$ with respect to the horizontal space variable $x$ and time $t, z$ being the vertical marching variable.

[25] J. F. Claerbout, Toward a unified theory of reflector mapping, Geophysics 36, 467 (1971).

[26] M. Frigo and S. G. Johnson, in Proceedings of the IEEE Vol. 93 (IEEE, 2005), p. 216.
[27] Complex operations are counted in terms of real additions and real multiplications.

[28] C. Tomas, L. Cazzola, D. Oriato, O. Pell, D. Theis, G. Satta, and E. Bonomi, in SEG Technical Program Expanded Abstracts 2012 (Society of Exploration Geophysicists, Tulsa, Oklahoma, USA, 2012), p. 1.

[29] The acquisition is specular to the phantom in Fig. 2.

[30] O. M. H. Rindal, A. Austeng, A. Fatemi, and A. RodriguezMolares, The effect of dynamic range alterations in the estimation of contrast, IEEE Trans. Ultrason. Ferroelectr. Freq. Control 66, 1198 (2019).

[31] W. C. Vogt, C. Jia, K. A. Wear, B. S. Garra, and T. J. Pfefer, Phantom-based image quality test methods for photoacoustic imaging systems, J. Biomed. Opt. 2, 1 (2017).

[32] The definition of $\sigma$ in the calculation of $C N R_{\mathrm{LC}}$ can be formulated either as the background noise, as in Eq. (12), or as the combined object-background noise [30]. The two definitions assume values in different ranges but are very close in the worst case (i.e., in images with low-contrast objects) [40].

[33] R. Wagner, S. Smith, J. Sandrik, and H. Lopez, Statistics of speckle in ultrasound b-scans, IEEE Trans. Sonics Ultrason. 30, 156 (1983).

[34] N. M. Ivancevich, J. J. Dahl, G. E. Trahey, and S. W. Smith, Phase-aberration correction with a 3-d ultrasound scanner: Feasibility study, IEEE Trans. Ultrason. Ferroelectr. Freq. Control 53, 1432 (2006).

[35] E. Bonomi, L. Brieger, C. Nardone, and E. Pieroni, Phase shift plus interpolation; a scheme for high-performance echo-reconstructive imaging, Comp. Phys. 12, 126 (1998).

[36] F. M. Hendriks, Mechanical Behaviour of Human Epidermal and Dermal Layers in Vivo (Technische Universiteit Eindhoven, Eindhoven, The Netherlands, 2005).

[37] P. Kruizinga, F. Mastik, N. de Jong, and A. F. W. van der Steen, Plane-wave ultrasound beamforming using a nonuniform fast fourier transform, IEEE Trans. Ultrason. Ferroelectr. Freq. Control 61, 2684 (2014).

[38] M. Mozumi and H. Hasegawa, Adaptive beamformer combined with phase coherence weighting applied to ultrafast ultrasound, Appl. Sci. 8, 1 (2018).

[39] T. Zhu, Time-reverse modelling of acoustic wave propagation in attenuating media, Geophys. J. Int. 197, 483 (2014).

[40] A. Konstantinidis, Comprehensive Biomedical Physics, A. Brahme (Editor in Chief), Physical Parameters of Image Quality (Elsevier, Amsterdam, The Netherlands, 2014). 